Symposium on Atomic \& Molecular Physics

\title{
DOUBLE PHOTOIONIZATION NEAR THRESHOLD
}

\author{
Ralf Wehlitz \\ Synchrotron Radiation Center, UW-Madison, Stoughton, WI 53589
}

\begin{abstract}
The threshold region of the double-photoionization cross section is of particular interest because both ejected electrons move slowly in the Coulomb field of the residual ion. Near threshold both electrons have time to interact with each other and with the residual ion. Also, different theoretical models compete to describe the double-photoionization cross section in the threshold region. We have investigated that cross section for lithium and beryllium and have analyzed our data with respect to the latest results in the Coulomb-dipole theory. We find that our data support the idea of a Coulomb-dipole interaction.
\end{abstract}

\section{INTRODUCTION}

The double-photoionization process, where the absorption of a single photon leads to the ejection of two electrons, is an interesting and also challenging subject in physics, because the breakup of a Coulomb system into three particles cannot be described analytically. Especially the threshold region, where both ejected electrons have time to interact with each other, has attracted the interest of theorists and experimentalists trying to find models of this seemingly simple process. Historically, interest has focused on a similar process, namely single ionization by electron impact, where - similar to the doublephotoionization process - two free electrons are in the final state, but leaving a singly charged ion behind. This interest was stimulated by its relevance for studying the gas discharge process.

In 1948 Wigner made a first attempt to describe the energy dependence of electron-impact ionization near threshold (ref. 1). This attempt lead to a linear law, i.e., the cross section is proportional to the energy above threshold (excess energy). Early experiments by Fox and Hickam et al. (refs. 2 and 3) seemed to support this law, even for the case of double ionization by electron impact.

Soon thereafter, Wannier examined the threshold region again and developed his famous threshold law (ref. 4). The idea is that in the asymptotic limit both emitted electrons have the same momentum (traveling on the "Wannier ridge") and are emitted back-to-back because of the Coulomb repulsion. If one electron would be faster than the other, the slower electron would be recaptured by the ion because of the missing shielding of the ion's potential by the fast electron. This leads to a power law for the double-electron escape at threshold, i.e., $\sigma \propto \mathrm{E}^{\alpha}$, with $\alpha$ being the Wannier exponent, which depends on the charge of the residual ion. For instance, for a neutral target, $\alpha=1.127$ for electron-impact ionization and $\alpha=1.056$ for double photoionization. In the extreme case where the residual ion has an infinite charge and the interaction between the electrons becomes negligible, one obtains again $\alpha=1.0$, which corresponds to the Wigner law.

Experimental evidence for $\alpha>1.0$ was obtained by McGowan and Clark for electron impact ionization of atomic hydrogen (ref. 5); they found $\alpha=1.13(3)$ for $\mathrm{E}<0.4 \mathrm{eV}$. An early photoionization experiment performed by Van der Wiel (ref. 6) produced data that were compatible with the Wannier's power law, but were not accurate enough to prove it. The first photoionization experiment that clearly supported Wannier's exponent was performed by Kossmann et al. (ref. 7) who found $\alpha=1.05(2)$ for double photoionization of helium. Samson et al. repeated the experiment (ref. 8) but used atomic oxygen instead of helium and found $\alpha=1.077(3)$.

Although the experiments mentioned above (refs. 5, 7, 8) are all in support of Wannier's theory, an alternate description of the electron double escape was developed by Temkin (see, e.g., ref. 9). This theory is based on the notion that, even near threshold, one electron is faster than the other and, thus, one electron has a larger distance from the ion than the other one. Therefore, the slower electron and the residual ion can form a dipole that rotates until the slow electron is "far away" from the ion. The fast electron experiences a field created by this Coulomb dipole and the cross section will exhibit a modulation, in contrast to the Wannier theory. The electron-impact cross section $\sigma$ can be described as follows: $\sigma \propto \mathrm{E}(\ln \mathrm{E})^{-2}[1+\mathrm{C} \sin (\alpha$ $\ln (E)+\mu)$ ], with $E$ the excess energy and $C, \alpha$ and $\mu$ suitable parameters. Note that the electron emission is 
not necessarily back-to-back anymore. It is worthwhile to mention that the formula given above can be applied to electron-impact ionization and double photodetachment because, in both cases, the residual ion and slow electron forms a dipole without a net charge. For double photoionization, however, this formula needs to be modified, because the "dipole" formed by the slow electron and residual ion has now a net charge.

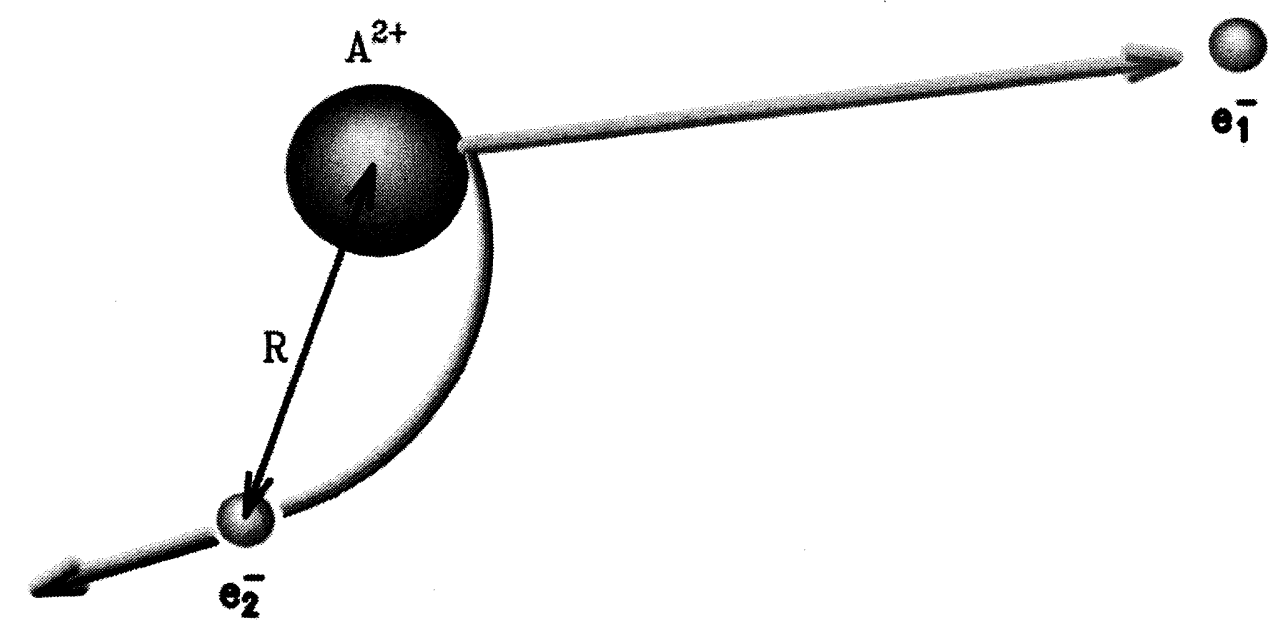

Figure 1 Sketch of the two electrons leaving a doubly charged ion behind after double photoionization.

Figure 1 illustrates the situation described above. While electron " 1 " is fast and far away, the slow electron " 2 " forms together with the ion a rotating dipole until it reaches a distance larger than " $R$ " and its path becomes a straight line. Experimental evidence that the Coulomb-dipole theory seems to be a realistic (measurable) model was given by Donahue et al. (ref. 10) studying double photodetachment of $\mathrm{H}^{-}$, and by Bae and Petersen (ref. 11) studying double photodetachment of $\mathrm{K}^{-}$. Although in both cases the cross section near threshold can be described by Wannier's power law, it is also possible to fit the formula of the Coulomb-dipole theory to their data. While the authors decided not to favor one of the theories, a detailed analysis performed later by Friedman et al. (ref. 12) indicates that the Coulomb-dipole theory provides a better modeling of the near threshold behavior of the cross section.

Considering the situation described above, we decided to investigate the double-photoionization cross section of lithium and beryllium near threshold. Up to that point, there was no clear evidence for the Coulomb-dipole theory, and double-photoionization experiments near threshold have been performed only for the elements $\mathrm{He}$ and $\mathrm{O}$. Our $\mathrm{Li}$ and Be data along with a preliminary fit model have been published recently (refs. 13, 14).

\section{EXPERIMENT}

The experiments were performed at the Synchrotron Radiation Center (SRC) in Wisconsin, which has an $800-\mathrm{MeV}$ electron storage ring that provides synchrotron radiation for various beamlines. The $\mathrm{Li}$ experiment was performed on the PGM Undulator beamline and the Be experiment was performed on the 4-m NIM beamline. Generally, the monochromatized photon beam enters the experimental chamber through a differential pumping stage. This pumping stage helps maintaining a very good vacuum in the beamline while working with gases in the experimental chamber. It also houses an array of filters that can be used to suppress second-order and stray light. The photon beam intersects an effusive beam of metal vapor that is created in a resistively heated furnace. The furnace can be electrically biased to prevent thermal electrons from reaching the interaction region. While thermal electrons do not have enough energy to ionize our target, the electric pulse applied across the interaction region, in order to extract the ions, is strong enough to accelerate these electrons to a sufficiently high kinetic energy. The ions extracted from the 
interaction region are accelerated into a drift tube and detected by a Z-stack of microchannel plates. This time-of-flight method allows us to separate the different charge states of our photoionized target atoms. Further details of the experiments can be found in references 13, 14, and 15.

\section{RESULTS}

After measuring the double-photoionization cross section of $\mathrm{Li}$, we applied the Wannier threshold law to our data and found an exponent $\alpha=1.054(7)$, which is close to the predicted value of 1.056. However, the difference between the fit curve and our data points exhibits a non-statistical modulation that reminded us on the modulation in the Coulomb-dipole theory. As mentioned above, a corresponding theory for double photoionization had not been developed at that time. However, Aaron Temkin provided us with a preliminary formula that fits nicely and even better to our data than the Wannier formula over $1.4 \mathrm{eV}$ above threshold (ref. 13). At this point we believed that the applicability of the (modified) Coulomb-dipole theory is due to the strong asymmetry in the Li atom. For double ionization near threshold, one electron is ejected from the $2 \mathrm{~s}$ shell while the other electron is ejected from the $1 \mathrm{~s}$ shell. The binding energy of the $2 \mathrm{~s}$ electron is only $5.4 \mathrm{eV}$ whereas the $1 \mathrm{~s}$ electron is tightly bound at $64.4 \mathrm{eV}$. Thus, $\mathrm{Li}$ is very different from He where both electron have the same binding energy.
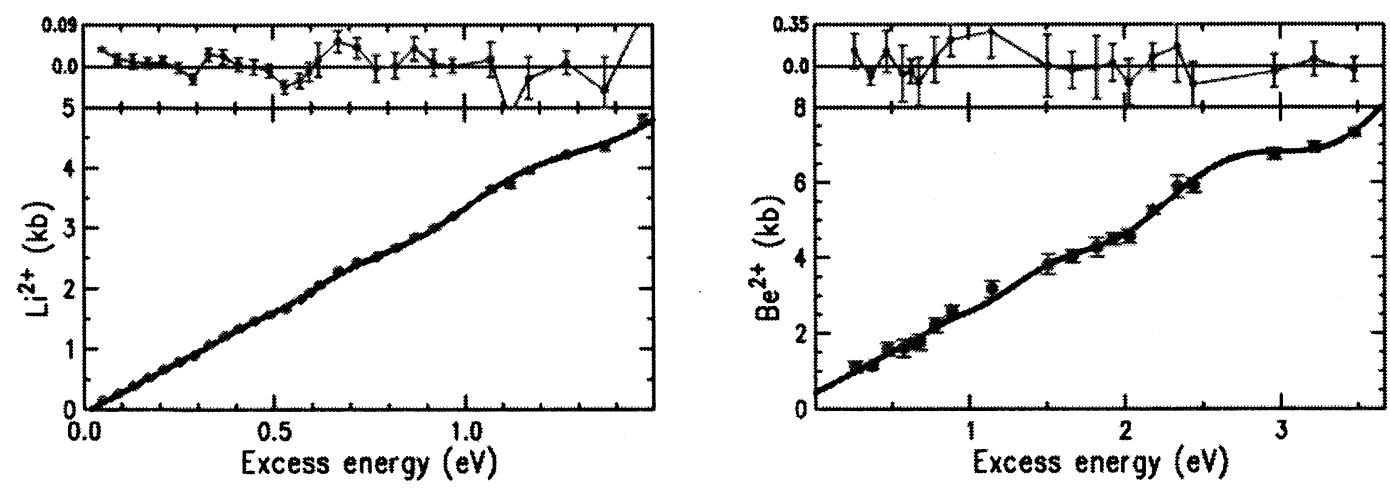

Figure 2 Double-photoionization cross section of $\mathrm{Li}$ (left) and $\mathrm{Be}$ (right) as a function of excess energy near threshold. The fit curves are according to Equation 1. The top panels show the difference of the fit curve from the data points.

In order to test this hypothesis we decided to measure the double-photoionization cross section of Be that is similar to He in so far as both outer s-electrons are emitted. The Wannier threshold law seemed to fit up to $1.8 \mathrm{eV}$ above threshold but, to our surprise, we found again an oscillatory behavior of the difference between our data points and the fit curve (ref. 14). A preliminary formula, suggested by Temkin, yielded again a better agreement with our data than the Wannier power law.

Only very recently Temkin and Bhatia ${ }^{1}$ found a formula that correctly describes the doublephotoionization cross section $\sigma$ near threshold within the framework of the Coulomb-dipole theory, namely:

$$
\sigma \propto \mathrm{E}+\mathrm{E}^{2} /(\ln \mathrm{E})^{2} * \mathrm{M}(\mathrm{E}), \text { with } \mathrm{M}(\mathrm{E})=\mathrm{B} \sin [\mathrm{A} \ln (\mathrm{E})+\mathrm{C}]
$$

Here, $\mathrm{E}$ is the excess energy and $\mathrm{A}, \mathrm{B}$, and $\mathrm{C}$ are suitable constants. From an experimental point of view the appearance of the new fit curve is only marginally different from the curves that we have used in the past. Unfortunately, the analytical expression is strictly valid only for the first $\sim 10^{-6} \mathrm{eV}$ above threshold, an energy range that will experimentally not be accessible in the near future. Drs. Temkin and Bhatia are in

\footnotetext{
${ }^{1}$ Private communication (2006)
} 
the process of applying their theory to the numerical evaluation of the cross sections in the experimental energy range, for the three targets $\mathrm{He}, \mathrm{Li}$, and $\mathrm{Be}$. This will provide a more stringent test of the theory than the parametric fit based on Eq. (1). Our Li and Be data along with the new fit curves are shown in Figure 2. In either case, the data displayed in the top panels show the difference between the cross section data and the fit curve. The fit curves seem to match the data very well since this difference exhibits just a random scatter of the points. One of the predictions of the Coulomb-dipole theory is the target independence of the parameter $\mathrm{A}$ in the equation above. From our fits we obtain values for the A parameter of 12.4(1) for $\mathrm{Li}$ and 12.1(10) for Be, which are in excellent agreement with this prediction.

Now the question arises: what about helium? No oscillations have been found in this case, neither experimentally (ref. 7) nor theoretically (ref. 16). Nevertheless, we applied the Coulomb-dipole theory to the He data and found a fit curve that is compatible with the He data. However, the agreement may be fortuitous and more precise data are called for. It will be of interest to see if the numerical calculations of Temkin and Bhatia will find a significant reduction in the magnitude of the oscillation for the He target compared to $\mathrm{Li}$ an $\mathrm{Be}$, as is require by the data.

It is worthwhile to note that in his 1982 paper (ref. 9), Temkin gives the approximate range for the A parameter, namely $7<\mathrm{A}<160$. Our A-parameter values (see above) as well as the values of Donahue et al. (ref. 10), $\mathrm{A}=42$, and $\mathrm{Bae}$ and Petersen (ref. 11), $\mathrm{A}=9.4$, are within the expected range.

\section{SUMMARY}

In order to test the Coulomb-dipole theory, we have determined the double-photoionization cross section of $\mathrm{Li}$ and $\mathrm{Be}$ near threshold. We have found oscillations in the cross section that are consistent with the recently developed Coulomb-dipole theory for double photoionization. Although this theory is, strictly speaking, only applicable for the first $\sim 10^{-6} \mathrm{eV}$ it provides a good description of the energy dependence of the experimental data. The A parameter that describes the "wavelength" of the oscillations is indeed target independent (for $\mathrm{Li}$ and $\mathrm{Be}$ ) as predicted and is of the expected order of magnitude.

While our data are in good agreement with the Coulomb-dipole theory, the rather large error bars do not allow us to make a final decision without some doubt. Although the three-body Coulomb interaction in the double-photoionization process is of fundamental importance, it is experimentally hardly accessible for two reasons. First, the cross section starts at zero at threshold resulting in very low count rates. Second, the "near threshold region" is not well defined and theory suggests that the threshold laws are applicable for only the fist $10^{-6} \mathrm{eV}$, an energy range that is not accessible by experiment at the moment. Of course, there is always hope that the theory is approximately valid for higher energies in the few-eV region.

I wish to thank Jaques Bluett, Dragan Lukic, and Scott Whitfield for their various contributions to the experiments. In particular, I want to thank Aaron Temkin for many stimulating discussions and for developing, together with Anand Bhatia, the Coulomb-dipole theory for double photoionization. The experiments were performed at the Synchrotron Radiation Center (SRC), which is supported by NSF under Grant No. DMR-0084402.

\section{REFERENCES}

1. E. P. Wigner, Phys. Rev. 73, 1002 (1948).

2. W. M. Hickam, R. E. Fox, and T. Kjeldaas, Jr., Phys. Rev. 96, 63 (1954).

3. R. E. Fox, W. M. Hickam, T. Kjeldaas, Jr., and D. J. Grove, Phys. Rev. 84, 859 (1951).

4. G. H. Wannier, Phys. Rev. 90, 817 (1953).

5. J. W. McGowan and E. M. Clarke, Phys. Rev. 167, 43 (1968).

6. M. J. Van der Wiel, Phys. Lett. 41A, 389 (1972). 


\section{Symposium on Atomic \& Molecular Physics}

7. H. Kossmann, V. Schmidt, and T. Andersen, Phys. Rev. Lett. 60, 1266 (1988).

8. Z. X. He, R. Moberg, and J. A. R. Samson, Phys. Rev. A 52, 4595 (1995).

9. A. Temkin, Phys. Rev. Lett. 49, 365 (1982).

10. J. B. Donahue, P. A. M. Gram, M. V. Hynes, R. W. Hamm, C. A. Frost, H. C. Bryant, K. B. Butterfield, D. A. Clark, and W. W. Smith, Phys. Rev. Lett. 48, 1538 (1982).

11. Y. K. Bae and J. R. Petersen, Phys. Rev. A 37, 3254 (1988).

12. J. R. Friedman, X. Q. Guo, M. S. Lubell, and M. R. Frankel, Phys. Rev. A 46, 652 (1992).

13. R. Wehlitz, J. B. Bluett, and S. B. Whitfield, Phys. Rev. Lett. 89, 093002 (2002).

14. D. Lukic, J. B. Bluett, and R. Wehlitz, Phys. Rev. Lett. 93, 023003 (2004).

15. R. Wehlitz, D. Lukic, C. Koncz, and I. A. Sellin, Rev. Sci. Instrum. 73, 1671 (2002).

16. U. Kleimann, T. Topçu, M. S. Pindzola, and F. Robicheaux, J. Phys. B 39, L61 (2006). 